\title{
TWIST1, A novel androgen-regulated gene, is a target for NKX3-1 in prostate cancer cells
}

\author{
Turid Eide ${ }^{1 *}$, Håkon Ramberg ${ }^{1}$, Carlotta Glackin ${ }^{3}$, Donald Tindall ${ }^{4}$ and Kristin Austlid Taskén ${ }^{1,2}$
}

\begin{abstract}
Background: TWIST1 plays a key role in EMT-mediated tumor invasion and metastasis. Since bone metastasis is a hallmark of advanced prostate cancer and is detected in at least $85 \%$ of patients who die of this disease, it is of great importance to understand the regulation of the cellular signaling pathways involved in the metastatic process.

Methods: Prostatic cell lines were analyzed using real time RT-PCR, chromatin immunoprecipitations (ChIP) and transfection of siRNA's and reporter constructs.

Results: We report in this paper that TWIST1 is an androgen-regulated gene under tight regulation of NKX3-1. Androgens repress the expression of TWIST1 via NKX3-1, which is a prostate-specific tumor suppressor that is downregulated in the majority of metastatic prostate tumors. We show that NKX3-1 binds to the TWIST1 promoter and that NKX3-1 over-expression reduces the activity of a TWIST1 promoter reporter construct, whereas NKX3-1 siRNA up-regulates endogenous TWIST1 mRNA in prostate cancer cells.
\end{abstract}

Conclusion: Our finding that NKX3-1 represses TWIST1 expression emphasizes the functional importance of NKX3-1 in regulating TWIST1 expression during prostate cancer progression to metastatic disease.

Keywords: Prostate cancer, LNCaP cells, TWIST1, NKX3-1

\section{Background}

Metastasis is the leading cause of morbidity and mortality among men with prostate cancer $(\mathrm{PCa})$. Metastasis is a complex multistep process controlled by distinct genes and signaling pathways in each step. Epithelial-meschencymal transition (EMT), a critical event for morphogenetic movements during formation of parietal endoderm during gastrulation, may represent the initial phase of metastasis. Furthermore, EMT seems to induce stem-like properties of epithelial cells [1].

A pivotal step of EMT is the loss of E-cadherin [2]. TWIST1, a master regulator of mesodermal development and a key mediator in the metastatic process, represses the expression of E-cadherin [3]. Furthermore, TWIST1 depletion reduces the expression of $\mathrm{N}$-cadherin in PC3 cells, a metastatic prostate cancer cell line, suggesting that TWIST1 supports EMT in prostate cancer [4]. In support of this, a positive correlation between the level of TWIST1 and prostate cancer metastasis has been reported [5].

\footnotetext{
* Correspondence: turid.eide@ous-hf.no

${ }^{1}$ Department of Tumor Biology, Institute for Cancer Research, Norwegian

Radium Hospital, Oslo University Hospital, Oslo, Norway

Full list of author information is available at the end of the article
}

NKX3-1 is a critical gene associated with early stage of prostate tumorigenesis as down-regulation of NKX3-1 is observed in both prostatic intraepithelial neoplasia (PIN) and adenocarsinomas [6]. Whereas high levels of TWIST1 is expressed in prostate cancer metastasis, low levels of NKX3-1 expression is observed in most prostate cancer metastasis examined [7]. NKX3-1 encodes a homeobox gene that is switched on during embryonic development of prostate tissue and is one of the earliest markers of luminal prostate epithelium [8]. NKX3-1 expression is strictly regulated by androgens and also appears to mark a sub-population of prostate stem cells $[9,10]$.

We report in this paper that TWIST1 is a novel androgen-regulated gene whose expression is tightly controlled by NKX3-1.

\section{Results}

TWIST1 is regulated by androgens

The expression of TWIST1 mRNA increased 4-fold in LNCaP cells incubated with $0.5 \mathrm{nM} \mathrm{R} 1881$ for 72 hours relative to untreated cells (ctr.) and reached more than a 10-fold induction at $10 \mathrm{nM}$ R1881 (Figure 1A). 


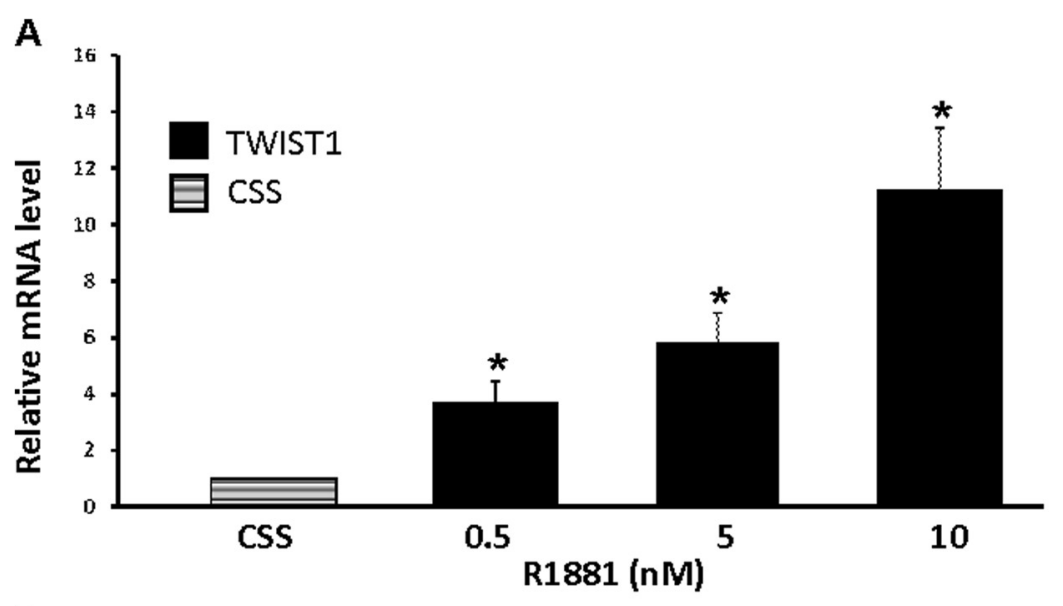

B

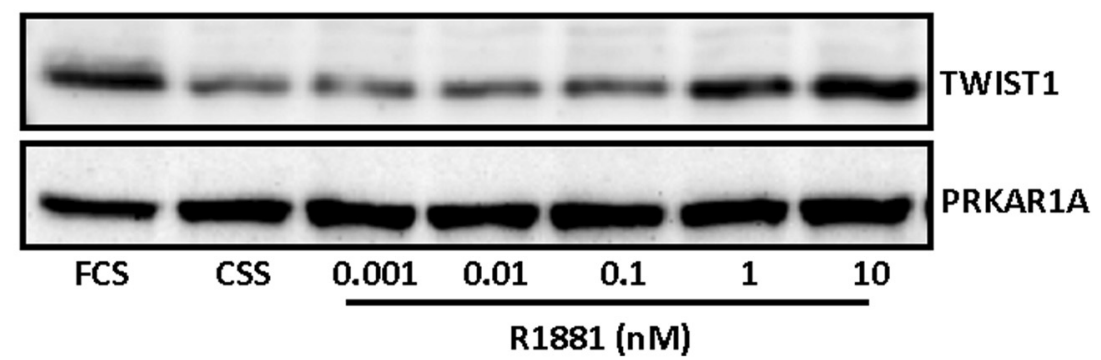

Figure 1 Androgen up-regulates TWIST1 expression. A. LNCaP cells were stimulated with increasing doses of the synthetic androgen R1881, and the relative level of TWIST1 compared to LNCaP cells left untreated in charcoal stripped fetal calf serum and phenol red free medium (CSS) was quantified using sqRT-PCR. Data are presented as mean $\pm S D(n=3)$ relative to unstimulated cells (control). A paired t-test was performed and a two-tailed p-value $<0,05$ is indicated with $a^{*}$. B. LNCaP cells were stimulated with increasing doses of the synthetic androgen R1881 or left untreated in CSS for 3 days before total protein was extracted. A representative Western blot probed with anti human TWIST1 antibody and anti PRKAR1A antibody as loading control is shown.

Corresponding regulation of TWIST1 mRNA was also demonstrated in RWPE-1, a benign prostate cell line (data not shown). Up-regulation of TWIST1 expression was also observed at the protein level in LNCaP cells stimulated with increasing concentrations of R1881 (Figure 1B).

To determine if AR mediates the effect of androgens on TWIST1 expression, LNCaP cells were transfected with a siRNA targeting AR (AR) or a non-silencing siRNA as control (ctr.). Down-regulation of AR expression (Figure 2, lower panel) reduced the mRNA level of TWIST1 by $80 \%$ relative to control (Figure 2A, upper panel) supporting the concept that TWIST1 is an AR target gene in LNCaP cells. Down-regulation of AR also reduced the level of NKX3-1 (Figure 2, lower panel).

\section{TWIST1 is an NKX3-1 target gene}

In an effort to find binding sites for AR (ARE) or androgen-regulated transcription factors, we examined the human TWIST1 genomic sequence using MatInspector [11]. Three putative binding sites for NKX3-1 designated BS1, BS2 and BS3 (Figure 3A) were identified. In addition we observed several potential AREs. Chromatin immunopreciptation analyses (ChIP) using chromatin isolated from LNCaP cells stimulate with $10 \mathrm{nM} \mathrm{R} 1881(+\mathrm{R} 1881)$ for 24 hours or unstimulated cells as control (-R1881) resulted in amplification of BS3. We were not able to detect binding to either BS1 or BS2 (data not shown, $\mathrm{n}=3$ ). A 5-fold increase in NKX3-1 binding activity to the TWIST1 promoter upon R1881 stimulation was observed (Figure 3B). Additionally, PCR amplification of non-targeted DNA (genomic contig of chromosome 12) was used as negative control (data not shown).

Next, a reporter construct containing $5 \mathrm{~kb}$ of the mouse TWIST1 promoter (pGL3-TWIST-Luc) was co-transfected with an NKX3-1 expression vector. Over-expression of NKX3-1 reduced the promoter activity of TWIST1 by $50 \%$, indicating that NKX3-1 represses the TWIST1 promoter activity (Figure 3C). No effect of NKX3-1 over-expression was observed on cells transfected with pGL3 lacking the TWIST1 promoter (pGL3).

Finally, the effect of NKX3-1 on endogenous TWIST1 mRNA level was studied by transfecting LNCaP cells with siRNA targeting NKX3-1. A 5-fold increase in TWIST1 mRNA level was observed when NKX3-1 expression was down-regulated (Figure 3D). The impact of R1881 stimulation, NKX3-1 overexpression and siRNA on NKX3-1 


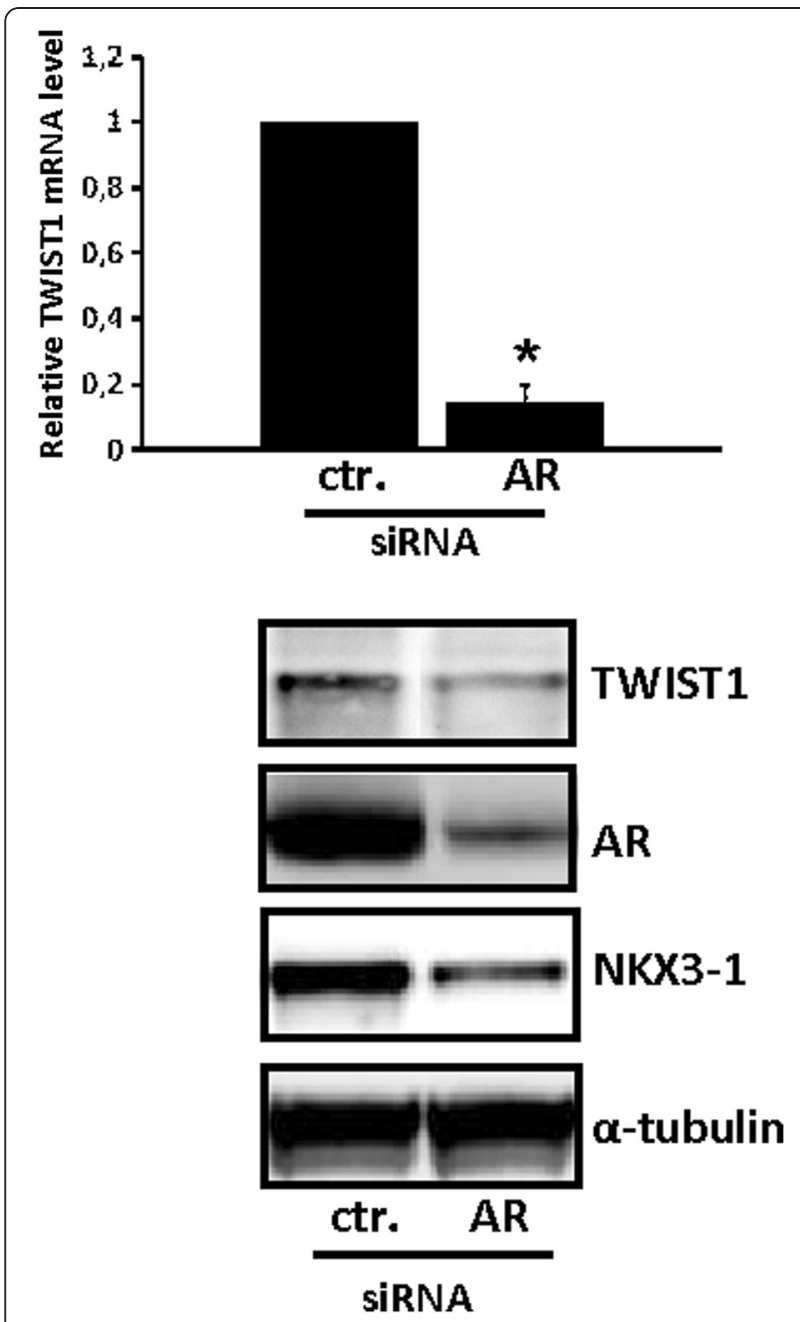

Figure 2 AR mediates the effect of androgen on TWIST1 expression. LNCaP cells cultured in RPMI medium containing 10\% FCS were transfected with $100 \mathrm{nM}$ of siRNA against AR (AR) or a non-silencing control (ctr.) siRNA. Total RNA and protein was harvested at $48 \mathrm{~h}$ after transfection. Upper panel: TWIST1 mRNA expression was examined using sqRT-PCR. Data are presented as mean $\pm S D(n=3)$ relative to the levels of $L N C a P$ cells transfected with a non-silencing control siRNA. A paired t-test was performed and a two-tailed $p$ value $<0,05$ is indicated with $a *$. Lower panel: Representative Western blots of TWIST1, AR, NKX3-1 and a-tubulin (loading control) are shown.

expression in LNCaP cells are summarized in Figure 3E. Both R1881 stimulation as well as overexpression of NKX3-1 led to a significant increase in NKX3-1 expression, while siRNA against NKX3-1 reduced the expression markedly.

\section{Discussion}

In this study we show both that TWIST1 mRNA is up-regulated by androgen via AR and that NKX3-1, a well-known androgen-regulated gene, binds the upstream regulatory region of the TWIST1 gene and represses the expression of TWIST1.

In a recent study, Takayama et al. [12] identified putative direct target genes of AR using ChIP-on ChIP and Cap Analysis Gene Expression (CAGE). One of the genes identified by in silico analysis was TWIST1. Our results support this report as increased levels of TWIST1 mRNA was observed in R1881 stimulated LNCaP cells, while reduced expression of TWISTl was observed in cells transfected with siRNA targeting AR.

Furthermore, our data shows binding of NKX3-1 to the promoter region of TWIST1. We therefore suggest that NKX3-1 mediates an indirect and late effect of androgen stimulation on TWIST1 expression. Interestingly, we show that siRNA targeting AR reduces the level of TWIST1, whereas siRNA targeting NKX3-1 increases TWIST1 expression suggesting that TWIST1 expression is tightly controlled by androgen. NKX3-1 has been shown to function both as an activator and a repressor of transcription, but few target genes have been identified [13-15].

The physical binding of NKX3-1 to the TWIST1 promoter might block the mesenchymal drive of TWIST1, until NKX3-1 expression is down-regulated or lost in PIN or adenocarcinoma lesions. Loss of NKX3-1 expression has been observed in $\sim 20 \%$ of PIN lesions, $\sim 40 \%$ of advanced prostate tumors and up to $80 \%$ of metastatic prostate cancer [16]. Androgen deprivation therapy as the most widely used treatment for advanced prostate cancer is likely to abolish androgen-stimulation of NKX3-1, leading eventually to down-regulation of repressor protein and de-repression of TWIST1's metastatic potential.

In an attempt to identify genes whose regulation are altered by NKX3-1, Song et al. [17] performed gene expression profiling analyses on micro dissected glands from NKX3-1-deficient prostate tissues during prostate cancer progression. They observed similarities between the expression profile of the micro dissected glands and constitutive activated AKT-transgenic mice as well as PTEN-deficient mice, suggesting that the PTEN-AKT-NKX3-1 axis serve as a major molecular path of prostate tumorigenesis. Li and Zhou [18] showed that activation of the AKT pathway by TWIST1 is critical for the sustention of cancer stem cell-like traits generated by EMT, again suggesting a link between loss of NKX3-1 expression, relive of TWIST1 expression and eventually activation of AKT pathway.

\section{Conclusions}

We report in this paper that TWIST1 is an androgenregulated gene, tightly regulated by NKX3-1. We show that NKX3-1 binds to the TWIST1 promoter and that NKX3-1 over-expression reduces the activity of a TWIST1 promoter reporter construct, whereas NKX3-1 siRNA upregulated endogenous TWIST1 mRNA in prostate cancer 


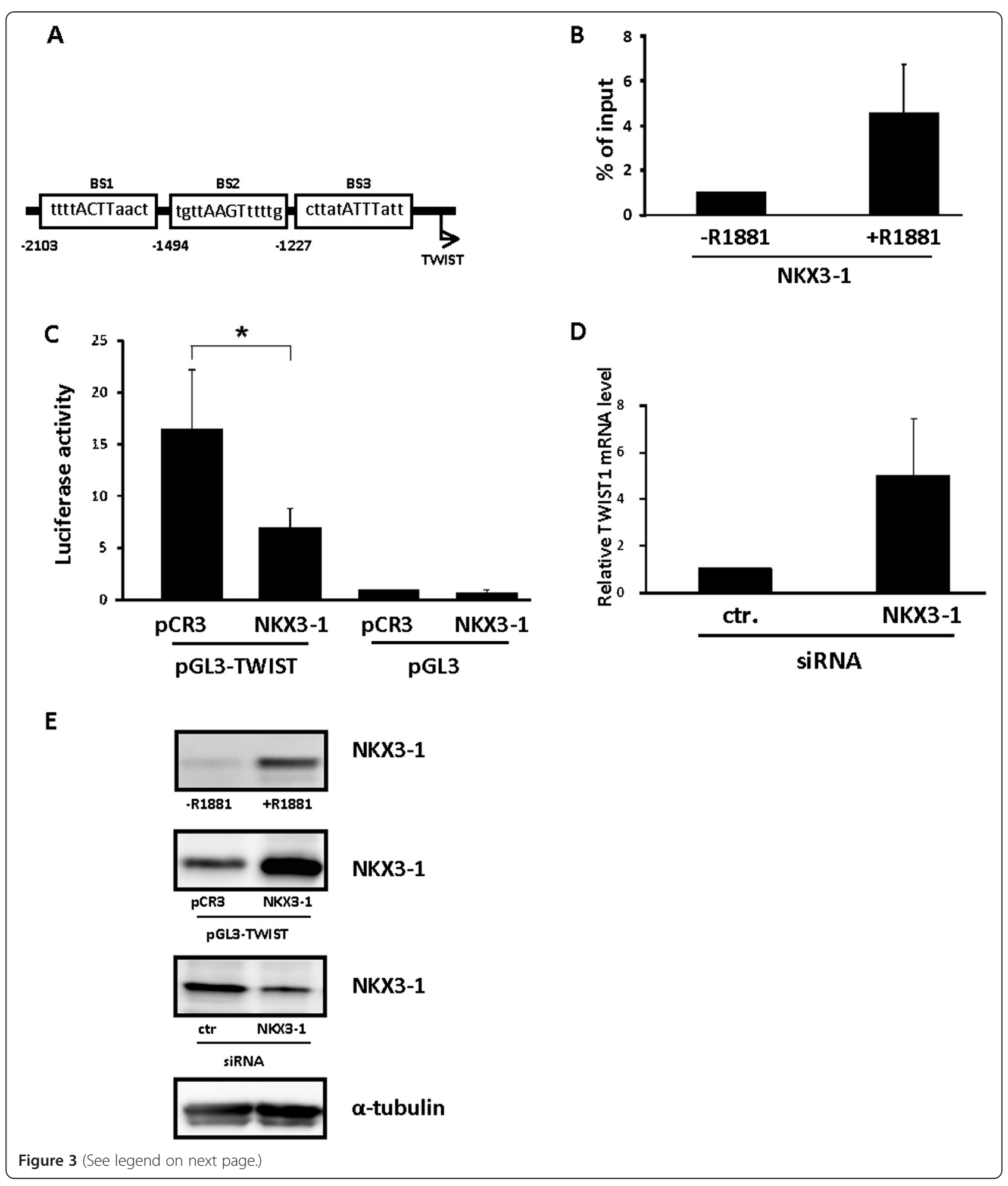




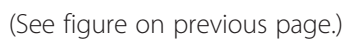

cells. Our finding that NKX3-1 represses TWIST1 expression emphasizes the functional importance of NKX3-1 in regulating TWIST1 expression during prostate cancer progression to metastatic disease.

\section{Methods \\ Cell culture}

LNCaP and RWPE-1 cells were purchased from ATCC (Rockville, MD) and cultured in RPMI 1640 medium containing 10\% fetal calf serum (FCS) or KeratinocyteSFM medium from Invitrogen (Carlsbad, CA, USA) supplemented with $2.5 \mu \mathrm{g}$ Epidermal Growth Factor (EGF) and $25 \mathrm{mg}$ Bovine Pituitary Extract (BPE), respectively, and stimulation with synthetic androgen R1881 was performed as previously described [19].

\section{Semi-quantitative real time RT-PCR (sqRT-PCR)}

Total RNA was isolated using Trizol ${ }^{\mathrm{TM}}$ from Invitrogen (Carlsbad, CA), and $100 \mathrm{ng}$ of total RNA was used in a one-step RT-PCR reaction (QIAGEN Quantitect SYBR Green RT-PCR kit) that was performed using an MJ Research DNA Engine Opticon Continuous Fluorescence Detection System (MJ Research Inc., Waltham, MA). RTPCR cycles were performed as previously described by Ramberg et al. [20]. G6PD was used for normalization. The $\Delta \Delta \mathrm{Ct}$ formula was used as described in the protocol from Applied Biosystems (Foster City, CA). All the PCRproducts were verified by sequencing.

\section{Primer sets used in sqRT-PCR}

G6PD (NM_000402); Left: tgcatgagccagataggc and right: acagggaggagatgtggttg, NKX3-1 (NM_006167); Left: gagacgctggcagagacc and right: ttctgcggctgcttaggg, AR (NM_000044); Left: gcgatccttcaccaatgtca and right: cattcggacacactggctgt, TWIST1 (NM_000474); Left: cttc tcggtctggaggatgg and right: ctccttctctggaaacaatgaca.

\section{Immunoblotting}

Protein extraction followed by Western blot analysis was performed as previously described by Kvissel et al. [19]. Primary antibodies used were the following: anti-TWIST1 (H-81, sc-15393), anti-AR (N-20, sc-816), both from Santa Cruz Biotechnology (Santa Cruz, CA). The anti-NKX3-1 antibody was kindly provided by Professor Fahri Saatcioglu at Department of Molecular Biosciences, University of Oslo, Norway. For loading control we used anti-PRKAR1A (610609, BD Transduction Laboratories) or anti- $\alpha$-tubulin antibody from Sigma (St. Louis, MO).

\section{Transfection and luciferase assay}

LNCaP cells were cultured at 300.000 cells per 6-well dish and transfected with a luciferase reporter plasmid including $5 \mathrm{~kb}$ of the mouse TWIST1 promoter- pGL3TWIST (kindly provided by Steven Kendall and Carlotta Glackin, Beckman Research Institute, City of Hope, USA) or pGL3 as negative control using Lipofectamine 2000 (INVT11668019, Invitrogen) according to the manufactures protocol. For overexpression of NKX3-1, a commercial transfection-ready TRUE clone (sc116287, ORIGENE, Rockville, MD, USA) was purchased. Cells were co-transfected with $\mathrm{pCMV} \beta$-gal (Clontech) to monitor the transfection efficiency. After 72 hours, luciferase and $\beta$-galactosidase activity were measured as previously described [20].

\section{Preparation and transfection of synthetic small interfering RNA (siRNA)}

siRNA targeting the following sequence within the androgen receptor mRNA: 5'-AAAAGCCCATCGTAGAG GCCCCA-3' was purchased from Dharmacon (Dharmacon Inc. (Lafayette, $\mathrm{CO}$ ). A non-silencing siRNA (cat.no D-001810-10-20, Dharmacon) was included in all siRNA experiments. siRNA against NKX3-1 was purchased from QIAGEN (2-for-silencing) and included the following sequences: NKX3-1-1: 5'CAGGCTATCATATATACTGTA -3', NKX3-1-2: 5'-ACGCTATAAGACTAAGCGAAA-3'. 
All siRNAs were transfected into cells using Dharma$F E C T^{\mathrm{TM}} 3$ transfection reagent according to the manufacture's protocol (cat.no T-2003, Dharmacon).

\section{Chromatin immunoprecipitation}

ChIP assays were carried out according to the QuickChIP protocol (Imgenex, San Diego, CA) with a crosslinking time of 10 minutes using $1 \%$ formaldehyde for the $\mathrm{LNCaP}$ cells and using an anti human NKX3-1 monoclonal antibody (cat.no 35-9700, Zymed Laboratories Inc., San Fransisco, CA). Following DNA purification (QuickChIP DNA Purification kit), sqPCR was performed using QIAGEN Quantitect SYBR Green RT-PCR kit with the following primers:

NKX3-1 BS3; Left: cccagttacacttggatgcagta and right: tcccctggtgagatcatacatac. Negative control primers from human chromosome 12 genomic contig; Left: atggttgc cactggggatct and right: tgccaaagcctaggggaaga. Chromatin immunoprecipitated with IgG was used as a negative control. The $\Delta \Delta \mathrm{Ct}$ formula was used as described in the protocol from Applied Biosystems (Foster City, CA).

\section{Abbrevations}

PCa: Prostate Cancer; EMT: Epithelial-Meschencymal Transition; PIN: Prostatic Intraepithelial Neoplasia; AR: Androgen Receptor; ChIP: Chromatin Immunopreciptation Analyses.

\section{Competing interests}

The authors declare that they have no competing interest.

\section{Authors' contributions}

TE took part in designing the study and conducted the experiments, collected and analyzed the data, and wrote the manuscript. HR conducted the Western blot analysis of androgen stimulation of TWIST1 and took part in reviewing of the manuscript. CG contributed with the mouse TWIST1promoter plasmid and took part in reviewing of the manuscript. DJT and KAT both conceived and coordinated the study, and participated in the discussion of all analyses and critical revision of the manuscript. All authors have read and approved the final draft of the manuscript.

\section{Acknowledgements}

We thank the Department of Urology at the Mayo Clinic and Foundation in Rochester, Minnesota, USA and Department of Urology at the Oslo University Hospital, Aker. Furthermore, we thank Lucy Smith (Mayo Clinic, Rochester, USA) for skilled technical assistance and Fahri Saatcioglu (University of Oslo, Norway)for providing us with anti human NKX3-1 antibody. This work was supported by The Norwegian Research Council, Health Region South East, The Norwegian Cancer Society, University of Oslo and Oslo University Hospital, Aker. DJT was supported by NIH grants CA121277, CA91956, CA125747 and the T.J. Martell Foundation.

\section{Author details}

${ }^{1}$ Department of Tumor Biology, Institute for Cancer Research, Norwegian Radium Hospital, Oslo University Hospital, Oslo, Norway. ${ }^{2}$ Faculty of Medicine, University of Oslo, Oslo, Norway. ${ }^{3}$ Division of Molecular Medicine, Beckman Research Institute of the City of Hope, Duarte 91010-3011CA, USA.

${ }^{4}$ Department of Urology, Mayo Clinic and Foundation, Rochester 55905MN, USA.

Received: 16 August 2012 Accepted: 29 January 2013

Published: 31 January 2013

\section{References}

1. Mani SA, Guo W, Liao MJ, Eaton EN, Ayyanan A, Zhou AY, et al: The epithelial-mesenchymal transition generates cells with properties of stem cells. Cell 2008, 133:704-715.

2. Birchmeier W, Behrens J: Cadherin expression in carcinomas: role in the formation of cell junctions and the prevention of invasiveness. Biochim Biophys Acta 1994, 1198:11-26.

3. Yang J, Mani SA, Donaher JL, Ramaswamy S, Itzykson RA, Come C, et al: Twist, a master regulator of morphogenesis, plays an essential role in tumor metastasis. Cell 2004, 117:927-939.

4. Alexander NR, Tran NL, Rekapally H, Summers CE, Glackin C, Heimark RL: Ncadherin gene expression in prostate carcinoma is modulated by integrindependent nuclear translocation of Twist1. Cancer Res 2006, 66:3365-3369.

5. Kwok WK, Ling MT, Lee TW, Lau TC, Zhou C, Zhang X, et al: Up-regulation of TWIST in prostate cancer and its implication as a therapeutic target. Cancer Res 2005, 65:5153-5162.

6. Bethel CR, Faith D, Li X, Guan B, Hicks JL, Lan F, et al: Decreased NKX3.1 Protein expression in focal prostatic atrophy, prostatic intraepithelial neoplasia, and adenocarcinoma: association with gleason score and chromosome 8p deletion. Cancer Res 2006, 66:10683-10690.

7. Gelmann EP, Bowen C, Bubendorf L: Expression of NKX3.1 In normal and malignant tissues. Prostate 2003, 55:111-117.

8. Bhatia-Gaur R, Donjacour AA, Sciavolino PJ, Kim M, Desai N, Young P, et al: Roles for Nkx3.1 In prostate development and cancer. Genes Dev 1999, 13:966-977

9. Prescott $J$, Blok $L$, Tindall DJ: Isolation and androgen regulation of the human homeobox CDNA, NKX3.1. Prostate 1998, 35:71-80.

10. Shen MM, Wang X, Economides KD, Walker D, Abate-Shen C: Progenitor cells for the prostate epithelium: roles in development, regeneration, and cancer. Cold Spring Harb Symp Quant Biol 2008, 73:529-538.

11. Quandt K, Frech K, Karas H, Wingender E, Werner T: MatInd and MatInspector: new fast and versatile tools for detection of consensus matches in nucleotide sequence data. Nucleic Acids Res 1995, 23:4878-4884.

12. Takayama K, Tsutsumi S, Katayama S, Okayama T, Horie-Inoue K, Ikeda K, et al: Integration of cap analysis of gene expression and chromatin immunoprecipitation analysis on array reveals genome-wide androgen receptor signaling in prostate cancer cells. Oncogene 2011, 30:619-630.

13. Carson JA, Fillmore RA, Schwartz RJ, Zimmer WE: The smooth muscle gamma-actin gene promoter is a molecular target for the mouse bagpipe homologue, mNkx3-1, and serum response factor. J Biol Chem 2000, 275:39061-39072.

14. Simmons SO, Horowitz JM: Nkx3.1 Binds and negatively regulates the transcriptional activity of Sp-family members in prostate-derived cells. Biochem J 2006, 393:397-409.

15. Abate-Shen C, Shen MM, Gelmann E: Integrating differentiation and cancer: the Nkx3.1 Homeobox gene in prostate organogenesis and carcinogenesis. Differentiation 2008, 76:717-727.

16. Bowen C, Bubendorf L, Voeller HJ, Slack R, Willi N, Sauter G, et al: Loss of NKX3.1 Expression in human prostate cancers correlates with tumor progression. Cancer Res 2000, 60:6111-6115.

17. Song H, Zhang B, Watson MA, Humphrey PA, Lim H, Milbrandt J: Loss of Nkx3.1 Leads to the activation of discrete downstream target genes during prostate tumorigenesis. Oncogene 2009, 28:3307-3319.

18. Li J, Zhou BP: Activation of beta-catenin and Akt pathways by twist are critical for the maintenance of EMT associated cancer stem cell-like characters. BMC Cancer 2011, 11:49

19. Kvissel AK, Ramberg H, Eide T, Svindland A, Skalhegg BS, Tasken KA: Androgen dependent regulation of protein kinase a subunits in prostate cancer cells. Cell Signal 2007, 19:401-409.

20. Ramberg H, Eide T, Krobert KA, Levy FO, Dizeyi N, Bjartell AS, et al: Hormonal regulation of beta2-adrenergic receptor level in prostate cancer. Prostate 2008, 68:1133-1142.

doi:10.1186/1475-2867-13-4

Cite this article as: Eide et al:: TWIST1, A novel androgen-regulated gene, is a target for NKX3-1 in prostate cancer cells. Cancer Cell International 2013 13:4 